\title{
EDUCAR EN FILOSOFÍA PARA SABER ESTAR EN LA REALIDAD JURÍDICA
}

\author{
Gregorio Rojas GonZÁlez \\ Universidad Católica de Colombia
}

\section{Resumen}

Una pedagogía que no se fundamente sobre la reflexión rigurosa de lo que es la educación en su relación con la persona humana y del saber corre el riesgo de dejar por fuera aspectos importantes de orden metodológico y teleológico que falsean la ordenación de la conducta de la persona humana que el educador, el educando y la sociedad deben hacer mediante actos de razón. Por lo anterior, se emprende la presente reflexión en tres secciones: la primera recoge el estudio del primer eje de formación para el abogado que se propone como fundamento del proyecto educativo del Programa de Derecho (PEP) de la Universidad Católica de Colombia, denominada eje de formación filosófica; en la segunda parte, en línea con el pensamiento de Aristóteles, se muestra una reflexión sobre la Filosofía como un saber fundado en causas últimas mediante un método específico lógico-racional; en la tercera sección, se propone un método pedagógico para el proceso de formación del estudiante de la ciencia del Derecho.

Palabras clave: educar, pedagogía, saberes, conocimiento, realidad, realidad jurídica, formación, ciencia del Derecho.

El autor: profesor de la Universidad Católica de Colombia. Correo electrónico: grgucc@hotmail.com

Recibido: 22 de noviembre de 2015; evaluado: 28 de enero de 2016; aceptado: 2 de febrero de 2016. 


\title{
EDUCATING IN PHILOSOPHY IN ORDER TO KNOW HOW TO BE IN LEGAL REALITY
}

\author{
Gregorio Rojas GonZÁlez \\ Universidad Católica de Colombia
}

\begin{abstract}
A pedagogy that is not based on a rigorous reflection on what is education in relation to the human being and knowledge will risk missing important methodological and teleological aspects that falsify the ordering of human behavior carried out by teachers, students, and society through acts of reason. Therefore, this article is divided into three sections: the first section examines the first axis of training for lawyers proposed as the foundation of the educational project in the Law Program (PEP) at the Universidad Católica de Colombia, the so-called axis of philosophical training. The second part, in line with the thinking of Aristotle, presents a reflection on Philosophy as a knowledge based on ultimate causes through a specific logical-rational methodology. The third section proposes a teaching method for the education of legal science students.
\end{abstract}

Keywords: Education, pedagogy, wisdoms, knowledge, reality, legal reality, training, legal science.

About the author: Professor at the Universidad Católica de Colombia. Email: grgucc@hotmail.com

Received: November 22, 2015; reviewed: January 28, 2016; accepted: February 2, 2016. 


\section{Introducción}

"Estudio es una palabra que designa aplicación intensa de la mente a algo".

Según Aristóteles, ${ }^{2}$ toda ciencia consiste en un conocimiento por las causas. Esta afirmación es actual, pues la Epistemología de la ciencia contemporánea parte del principio de que el conocimiento científico es causado y es por ello que participa de la sabiduría, pues "llamamos el más sabio -sophos- en toda ciencia a aquel que puede dar razón de las causas de todo lo investigado, y por eso enseñar". ${ }^{3}$ También es propio del sabio ordenar las cosas a su fin, porque quien ignora el sentido último de las cosas termina por trastocar las cosas mismas y no acierta en sus acciones.

En esta línea, Tomás de Aquino se refiere a cuatro tipos de sabiduría, las cuales remiten a cuatro clases de orden. La primera, por ser de orden natural -filosofía natural- no ordena nada, ya que este orden no depende de la razón humana; la segunda corresponde a la lógica -filosofía racional- por estar dirigida a ordenar la actividad de la razón; la tercera consiste en la ordenación que la razón hace -filosofía moral- de los actos de la voluntad y la cuarta se dirige a ordenar las cosas exteriores -artes productivas-. Así pues, quien conoce las causas está habilitado para ordenar y el acto propio mediante el cual se ordenan las cosas por sus causas es lo que se denomina conocimiento científico o filosófico. ${ }^{4}$

Por lo expuesto, he abordado la presente reflexión en tres momentos. El primero recoge al pie de la letra el estudio del primer eje de formación para el abogado que se propone como fundamento del proyecto educativo del Programa de Derecho (PEP) de la Universidad Católica de Colombia, llamado eje de formación filosófica; en el segundo, en línea con el pensamiento de Aristóteles, se reflexiona sobre la Filosofía como un saber fundado en causas últimas mediante un método específico lógico-racional; en el tercero, se propone un método pedagógico para el proceso de formación del estudiante de la ciencia del Derecho.

Tomás de Aquino, Suma de Teología, parte II, 166: Templanza, La estudiosidad (Madrid: Biblioteca de autores cristianos, 2001), 551.

Aristóteles, Analíticos posteriores (Madrid: Gredos, 1994), 16.

Aristóteles, Metafísica (Madrid: Gredos, 1994), 70.

Enrique Martínez García, Persona y educación en santo Tomás de Aquino (Madrid: Fundación Universitaria Española, 2002), 16-17. 


\section{Eje de formación filosófica}

La Universidad Católica de Colombia y su Programa de Derecho, en desarrollo de la legislación y de uno de sus compromisos, según el cual en la educación ${ }^{5}$ intervienen la inteligencia y la libertad -y por ende es de naturaleza moral-, abordó entre los años 2001 y 2003 la revisión y el estudio de la estructura y de los contenidos del programa de Derecho. Con ello se buscó la formulación de una clara opción curricular y la propuesta de su respectivo plan de estudios para la formación del profesional del Derecho a la luz de la misión institucional, del proyecto educativo institucional (PEI), de su proyecto educativo del programa (PEP) y de su correspondiente estatuto epistemológico desde el realismo jurídico clásico. ${ }^{6}$

En desarrollo de ese cometido, los directivos de la Universidad y del Programa, así como un grupo de profesores, emprendieron la reflexión para determinar los ejes sobre los cuales habría de estructurarse el saber jurídico, su estatuto epistemológico, su opción curricular y su articulación con la misión institucional, el proyecto educativo institucional (PEI) y el proyecto educativo del programa (PEP). Su propósito era fijar la especificidad de formación jurídica que habría de ofrecer el programa

5 Por educar entendemos toda actividad que permite el desarrollo de las facultades intelectuales y morales de una persona para facilitar su inmersión en la realidad y la obra consecuente con ella. Estas ideas y otras más recogidas en este escrito han sido tomadas de diferentes conferencias pronunciadas por el exrector de la Universidad, doctor Edwin de Jesús Horta Vásquez. Universidad Católica de Colombia, "Derecho", https:// www.ucatolica.edu.co/portal/programas/programas-de-pregrado/derecho/ (acceso octubre 25, 2015).

6 Escuela de pensamiento filosófico propuesta por Aristóteles y Tomás de Aquino, cuyo fundamento es el sistema de pensamiento filosófico denominado realismo filosófico o realismo metódico. Si aplicamos lo que significa el realismo y el idealismo a la realidad que subyace en el concepto de Derecho, existirín dos respuestas a la pregunta sobre qué es el Derecho y cuál es su fundamento. La primera respuesta es que el Derecho es algo diferente a un acto de razón o una obra de conciencia y que se encuentra en una realidad distinta de lo meramente material. La otra respuesta sería que el Derecho es una realidad de razón o de conciencia y pertenece a las obras humanas y al mundo material. La primera posición define al Derecho como la cosa justa o como lo justo, en tanto la segunda lo define, de manera equivocada, como la ley o norma. El vocablo "realismo" se deriva de las palabras "real" y "realidad", procedentes del latín res (cosa). En el campo filosófico, el realismo designa las actitudes que, en distintos planos de la vida humana, subrayan el de las cosas por sí mismas, la primacía de lo real, entendiendo por tal lo "en-sí", con anterioridad y al margen de la relación cognoscitiva y operativa del hombre con las cosas.

"En otras palabras, el realismo consiste en la afirmación de una realidad que existe con independencia del sujeto pensante. Los objetos tienen una existencia diferente al pensamiento del sujeto que conoce, y tales objetos son cognoscibles (susceptibles de ser conocidos), además, son lo que ellos son en sí mismos y no lo que el sujeto conoce de ellos".

Dentro de las características del realismo filosófico encontramos que: a) las cosas existen fuera e independientemente de la conciencia o del sujeto pensante; b) conocemos como reales aquellas cosas que nos llegan por medio de los sentidos, y c) la existencia del mundo exterior es una evidencia que se manifiesta por la intuición sensible que se convierte en un juicio del sujeto que conoce. Gran enciclopedia Rialp, "Realismo" (Madrid: Rialp, 1989), 721-723. 
de Derecho de la Universidad Católica de Colombia y su correspondencia con las exigencias permanentes de justicia -no una justicia ideal, sino real-, en tanto son exigencias del espíritu humano y de la sociedad.

De tal reflexión surgió la propuesta inicial de fundamentarlo sobre tres pilares, que son:
a) Eje de formación filosófica.
b) Eje de formación jurídica o en justicia.
c) Eje de formación legislativa.

A estos tres ejes se suma otro, denominado "de formación sociojurídica".

En el eje de formación filosófica se pretende que el estudiante conozca los sistemas de pensamiento filosófico que fundamentan las escuelas jurídicas y demás teorías del Derecho, para que el futuro profesional tenga la suficiente capacidad argumentativa y opte críticamente en lo que se refiere a la fundamentación del Derecho desde la Filosofía y la metafísica que, en palabras de Zubiri, ${ }^{7}$ se concibe como el estudio de las cosas reales en tanto que reales, es decir desde el estudio o aprehensión de la realidad. ${ }^{8}$

Este estudio, que parte de considerar el Derecho como una realidad, proporciona los elementos suficientes para identificar los objetos de conocimiento básicos de la Ciencia Jurídica, a saber: la conducta humana -objeto material- y lo justo -objeto formal-, los cuales se ordenan a la justicia como fin último del saber jurídico. Estos saberes, a su vez, constituyen la naturaleza y estructura de la ciencia del Derecho como un conocimiento teórico y práctico y, como lo anota el profesor Javier Hervada, permiten definir y delimitar este Derecho, poner de relieve su estatuto

\footnotetext{
Xavier Zubiri, Cinco lecciones de Filosofía (Madrid: Alianza, 1982), IX.

3 El término realidad procede del latino res, que en primer lugar significa "cosa". Para los juristas romanos, res no solo abarca las cosas materiales, sino también entes inmateriales como el derecho de propiedad. De res deriva realis, real, a partir del cual se forma el vocablo abstracto "realidad", común en las lenguas modernas, pero inexistente en latín. Según su procedencia etimológica, en primer lugar debía significar la esencia de la cosa, aquello por lo que la cosa es tal, pero como otros vocablos abstractos (humanidad, por ejemplo), este se ha matizado como colectivo, por lo que, en el lenguaje ordinario, se utiliza para designar el conjunto de las cosas; así, equivale a "lo real", la totalidad de lo que es. En este amplísimo sentido, entendemos no solo el conjunto de los entes materiales, sino también el de los seres espirituales, las ideas, los valores, las sustancias. Gran enciclopedia Rialp, "Realidad" (Madrid: Rialp, 1989), 719.
} 
epistemológico y marcar los caracteres que lo diferencian de otras disciplinas, al igual que establecer el orden de las relaciones entre estas. ${ }^{9}$

\section{La Filosofía como conocimiento por causas últimas}

El profesor Xavier Zubiri ${ }^{10}$ destaca el conocimiento filosófico como el punto más alto del conocimiento causado que aborda las últimas y no las primeras causas y proporciona un tipo de saber que permite usos diferentes a los del saber científico; de ahí que se denomine también saber metafísico. ${ }^{11}$ Por esta razón, la Filosofía se relaciona estrechamente con la sabiduría:

Filosofía significa el gusto, el amor de la sabiduría y del conocimiento, sobre todo ese conocimiento que se logra por el examen o inspección de las cosas; un examen que los griegos llamaron theoria. Estos tres conceptos -filosofía, sofía y teoría- estuvieron siempre íntimamente asociados en la mente griega. Así, Heródoto atribuye a Creso estas palabras con que saluda a Solón: Han llegado hasta nosotros muchas noticias tuyas, tanto de tu sabiduría como de tus viajes, y de que, movido por el gusto del saber, has recorrido muchos países para examinarlos. ${ }^{12}$

Luego, para el mundo griego, el quehacer filosófico es un modo de conocimiento ${ }^{13}$ que consiste en una forma específica de relación entre el intelecto y lo que está fuera de él: la realidad. Esta realidad no siempre se manifiesta de una manera inequívoca, ya que el intelecto puede errar en el juicio, en el raciocinio o en la conclusión de lo real y generar en el sujeto una apariencia de lo real. Para hacer que el intelecto no falle en sus conclusiones, debe hacer uso de un método específico que le permita verificar la realidad seleccionada desde un objeto formal y material de estudio. ${ }^{14}$

Javier Hervada, Lecciones propedéuticas de Filosofía del Derecho. 3a edición (Pamplona: Eunsa, 2000), 119. Zubiri, Cinco lecciones de Filosofía, 27.

11 La palabra metafísica no se encuentra en los escritos de la obra de Aristóteles; es una denominación editorial dada por Andrónico de Rodas a una colección de escritos sin título que venían después de la Física, tà metà tà physikà.

12 Zubiri, Cinco lecciones de Filosofía, 12. En esta misma línea, puede verse Hervada, Lecciones propedéuticas, 4.

13 El fin del conocimiento es ordenar al ser humano en la realidad.

14 Estas ideas fueron pronunciadas por el doctor Edwin de Jesús Vásquez en la conferencia dictada en la Universidad Militar Nueva Granada, a propósito de las características del conocimiento filosófico, en abril 24 de 2013. 
Esta dimensión de la Filosofía como un saber privilegiado ha sido destacada por Aristóteles, desde el inicio de su metafísica, con las siguientes palabras: "Todos los hombres, por naturaleza, desean saber" ${ }^{15}$ Respecto a esta afirmación hecha por el estagirita, Frederick Copleston manifiesta:

[...] hay diferentes grados del saber. Por ejemplo, el hombre meramente experimentado, como Aristóteles dice, puede "saber" que cierta medicina le ha sido beneficiosa a X cuando estaba enfermo, pero sin llegar a saber el porqué de tal beneficio, mientras que el hombre del arte sabe la causa: conoce, supongamos, que $\mathrm{X}$ estaba febril y que la medicina en cuestión tiene cierta propiedad que hace desaparecer la fiebre. Este "entendido" conoce un universal, porque sabe que ese medicamento tenderá a curar a cuantos padezcan esa enfermedad. El arte, por consiguiente, se ordena a una producción de cierta clase, pero no es, en opinión de Aristóteles, la sabiduría, ya que la suprema sabiduría no se ordena a producir cosa alguna ni a asegurar ningún efecto -no es utilitaristasino que trata únicamente de averiguar los primeros principios de la realidad, es decir, trata de alcanzar el conocimiento por lo que este es en sí mismo. Aristóteles pone al hombre que busca el saber por lo que el saber mismo es en sí por encima del hombre que quiere adquirir un conocimiento particular con miras a lograr algún efecto práctico. En otros términos, es superior aquella ciencia que es deseable por sí misma y no sólo con miras a sus resultados. ${ }^{16}$

Afirma Aristóteles que, por naturaleza, el hombre y los animales retienen y mantienen lo que han sentido; el hombre, además, desea saber y saber es, por antonomasia, "poseer intelectivamente la verdad de las cosas". ${ }^{17}$ El hombre tiene modos de saber exclusivos y, por lo tanto, son de distinta índole a los de los animales, aunque también están apoyados en la experiencia organizada por la memoria. Estos modos de saber exclusivos del hombre son tékhne, phronesis, episteme, nous y sophia.

La tékhne, traducida por los latinos con el término arts arte, en castellano, hace referencia a la capacidad de saber hacer, producir o fabricar algo. La constituye el saber hacer algo en la verdad de las cosas, no la mera producción. Hay una diferencia entre el modo de saber experimental y la tékhne: con la experiencia se logra producir los efectos que se intentan y con la tékhne se sabe cómo conseguir el resultado esperado y el porqué de su producción. Por lo tanto, para Aristóteles, el modo de

\footnotetext{
Aristóteles, Metafísica, 75.

Frederick Copleston, Historia de la Filosofía 1: Grecia y Roma (Barcelona: Ariel SA, 1999), 160-161.

Aristóteles, Analíticos posteriores (Madrid: Gredos, 1994), 20.
} 
saber tékhne es superior al de la experiencia. ${ }^{18}$ En palabras más elaboradas, lo propio de la tékhne es saber el porqué de las cosas, su ser y "su razón de ser", lo que hace del saber algo universal y, por ello, superior al conocimiento experimental. Quien maneja la tékhne es el que mejor sabe comunicarla y enseñarla a los demás. ${ }^{19}$

El modo de saber de la tékhne o el uso práctico de la razón tiene una triple superioridad frente al conocimiento experimental, consistente en saber mejor, saber más y saber enseñar. El hombre que tiene la costumbre de obrar de acuerdo con el conocimiento práctico es un sophós, es decir, es un hombre que tiene el hábito de hacer las cosas con fundamento en la verdadera razón de ellas.

Por ello, a quien suele actuar así se le llama hombre prudente -del griego phrónesis, que en castellano significa prudencia-, en tanto la phrónesis concierne a las acciones poiesis que el hombre ejecuta sobre las cosas o sobre sí mismo praxis. Zubiri la definiría como "saber la manera de actuar en la vida en su conjunto total". ${ }^{20}$ En términos aristotélicos, es "una habitud de praxis con razón verdadera acerca de lo bueno y lo malo para el hombre". ${ }^{21}$ Por lo tanto, la phrónesis es un saber universal que tiene un objeto preciso: que el hombre pueda y sepa actuar bien o mal. A este orden de la Filosofía corresponde el conocimiento jurídico práctico como ciencia de la conducta humana en torno a lo que significa lesión a los derechos de la persona.

Intrínsecamente relacionados con estos modos de saber encontramos otro de rango superior: la episteme, también llamada "ciencia" por Aristóteles, que nos muestra las causas de las cosas y nos hace saber con verdad la necesidad constitutiva de las mismas. Es un modo de saber demostrativo que solo recae sobre lo que es. Esta demostración o exhibición que la cosa hace de su estructura interna acontece en un acto mental preciso: la lógica. De tal manera, los silogismos -premisas que conllevan una conclusión- conducen a la demostración, es decir, a la episteme.

La episteme en tanto ciencia -esto es, conocimiento concreto de un objeto por causas próximas- nos muestra la cosa de manera limitada y, por lo tanto, no abarca una demostración universal y se apoya en principios que son supuestos primarios

18 Al respecto, Xavier Zubiri formula el siguiente ejemplo: "El empírico -que se basa en la experiencia- sabe que Fulano está enfermo y que solicitándole tal fármaco se curará. En cambio, el que tiene tékhne sabe por qué se cura". Zubiri, Cinco lecciones de Filosofía, 19.

19 En esta misma línea, puede verse Ricardo Yepes Stork y Javier Aranguren, Fundamentos de Antropología: un ideal de la excelencia humana (Pamplona: Eunsa, 1998), 157.

20 Zubiri, Cinco lecciones de Filosofía, 21.

21 Zubiri, Cinco lecciones de Filosofía, 21. 
que, en la cosa misma, constituyen la base de su necesidad; al principio del conocimiento epistémico es a lo que el profesor Zubiri llama el nous. "El nous es ese modo de saber por el que, en forma videncial, inmediata e innata, aprehendemos las cosas en su ser inconmutable, que debe ser supuesto de la episteme" y, por lo tanto, la ciencia no puede renunciar a él. ${ }^{22}$

De lo anterior se desprende que la ciencia y los principios -episteme- y nous están en una especie de relación intrínseca que los conduce a la unidad y nos aproxima a la descripción que hace Carlos Cardona de la unidad del conocimiento. Podríamos decir que no es solo el sentido el que conoce y tampoco el intelecto de manera aislada, sino que el que conoce es todo el hombre por medio de sus sentidos y su intelecto. El intelecto parte de lo aportado por los sentidos y otros modos de saber y se pregunta por los primeros principios o por las últimas causas, por el sentido mismo de las cosas y de la realidad de estas en tanto realidad. A la unidad resultante la denomina sabiduría o sophia. En otras palabras, el modo de saber sophia es la interna unidad de nous y de episteme en el saber. Zubirí lo define como "el saber integral de algo, porque sólo entonces sabremos por completo lo que es el ser que necesariamente es siempre". ${ }^{23}$ A diferencia del episteme científico, que responde a cosas concretas y por razones o causas mediatas, el episteme filosófico responde a realidades universales y por últimas causas; la primera consecuencia que se deriva de ello es que el episteme filosófico responde por realidades que permanecen siempre y de manera necesaria, como la justicia o la libertad, realidades ante las que la ciencia no totalizante no puede responder.

Por ello, para Aristóteles, la Filosofía como modo de saber debe ser una ciencia que explique los principios, cuyo objeto universal, en el sentido de los conceptos y de abarcar la totalidad ${ }^{24}$ de las cosas, es el ente como tal a partir de la sustancia misma. En este sentido, la ciencia filosófica tiene que buscar las razones y las causas del ente que le pertenecen por su intrínseca razón de entidad. Filosofía, entonces, es saber qué son nous y episteme, esto es, una sabiduría, el más alto modo de saber humano.

Zubiri, Cinco lecciones de Filosofía, 22.

Zubiri, Cinco lecciones de Filosofía, 22.

4 Explica Zubiri, desde la filosofía aristotélica, que "la totalidad consiste en la coincidencia de todas las cosas en un mismo carácter". Zubiri, Cinco lecciones de Filosofía, 29. 


\subsection{La Filosofía como función intelectual}

Con base en su estudio del pensamiento aristotélico, el profesor Zubiri concluye que la Filosofía es un modo de saber humano; de tal manera, desempeña una función intelectual según la cual el que la posee -la Filosofía- en el sentido de que tiene como hábito desarrollarla con verdadera razón, es un sophós. ${ }^{25}$ La filosofía es un modo de saber peculiar que Aristóteles llamó ciencia apodíctica del ente en tanto tal, porque le proporciona al ser humano la capacidad de un saber especial para ubicarse en la realidad con pretensiones de sentido ante esta. ${ }^{26}$

En esta línea de argumentación, para Aristóteles, el propósito genérico del saber es hacer de quien lo posee un sophós, en el entendido de que este es "un hombre que posee una forma superior del saber, que puede ir desde el dominio de cualquier tékhne elemental hasta el saber del Universo, de la moral y de la política". ${ }^{27} \mathrm{El}$ sophós fue caracterizado por el estagirita de la siguiente manera:

a) Conoce todo aquello a lo que su saber se refiere, es decir, domina las cosas que le conciernen.

b) Domina el saber más difícil e inaccesible.

c) Posee un saber estricto, riguroso y exacto de las cosas.

d) Sabe las cosas en forma tal que puede enseñar a los demás lo enseñable lo máthema.

e) Su saber le confiere un carácter de rector, magisterio y mando de los demás.

f) Su saber no va ordenado a ninguna producción, sino que es una acción que se basta a sí misma. Por lo tanto, busca un saber no solo por los resultados, sino por el saber.

Sostiene el profesor Zubiri que "el hombre tiene en su vida distintos tipos de saber, que responden a distintas actividades suyas". ${ }^{28}$ En este sentido, el saber del hombre depende de su ocupación, de la cual se derivan preocupaciones en forma de interrogantes que, dada su naturaleza racional, son resueltos con saber. El primer tipo de saber es aquel al que el filósofo citado llama "zona de las urgencias". En este estadio, el hombre tiene que forzar su mente con el objeto de saber lo necesario y

\footnotetext{
Zubiri, Cinco lecciones de Filosofía, 31.

Aristóteles concibió la Filosofía como la ciencia apodíctica del ente en cuanto tal, es decir, como la ciencia que explica acerca de los principios de la sustancia misma. Zubiri, Cinco lecciones de Filosofía, 32.

Zubiri, Cinco lecciones de Filosofía, 38.

28 Zubiri, Cinco lecciones de Filosofía, 39.
} 
urgente para la vida -este tipo de saber es una tékhne-. En un segundo momento, resuelto lo urgente de la vida mediante el saber, el racional busca satisfacer sus necesidades apremiantes concernientes al placer, a la comodidad y a la vida fácil mediante lo que los latinos llamaron negotium, ya que produce utilidad. En una tercera etapa, el hombre se ocupa del ocio otium ${ }^{29}$ que produce el theorein y la theoría teoría que es la forma suprema de la praxis. Entonces, la teoría no nace de una necesidad vital y esto lo prueba que el motor que llevó al hombre a saber fue la admiración socrática ${ }^{30}$ característica del ocio. Toda ciencia es un modo de considerar y de inspeccionar el objeto de lo desconocido, de lo admirado y este saber es el que hace el hombre un sophós cuando logra dominarlo y, de esta manera, puede enseñar y conducir a los demás.

Ahora bien: en el ocio se ha originado también esa forma de saber que, en cierto modo, también es theoría: el mito. Consciente de esto, Aristóteles determina que el saber del que se ocupa es aquel que busca diseñar un conocimiento demostrativo del ente en cuanto tal. Se trata de esa ciencia que estudia la totalidad de las cosas en tanto son, justamente la sophía que le corresponde al filósofo, la sabiduría de primera línea de la que habla Zubiri, es decir, la Filosofía frente a la cual todas las formas del saber que antecedieron al gran filósofo estagirita no son más que sabidurías de segundo orden. Así, la Filosofía es sabiduría primaria en un doble sentido: porque versa acerca de lo supremo de las cosas -acerca de su entidad- y porque es la forma suprema de sabiduría. Esta sería la función intelectual de la ciencia filosófica: ser suprema sabiduría.

La anterior afirmación fue comprobada por Aristóteles mediante silogismos que relacionaban los caracteres del sophós griego y los de la ciencia apodíctica del ente como tal -Filosofía-, a saber:

a) Si el sophós es el que tiene un saber de la totalidad de su dominio, el que posee la ciencia filosófica del ente en tanto tal tiene que saber todo lo que esta ciencia abarca y su índole. Entonces, la Filosofía es un saber absoluto pues en el ser

29 En la Grecia de Aristóteles, el ocio tenía un significado distinto al actual, pues no consistía en no hacer nada, sino estudiar para lo no necesario y lo no negocioso. En términos de Xavier Zubiri, "no ir a las cosas, sino por las cosas mismas, saber de ellas por sólo lo que ellas son". Zubiri, Cinco lecciones de Filosofía, 40.

30 Por admiración socrática entendemos "la admiración que embarga al hombre que cree saber perfectamente aquello de lo que se ocupa, cuando un buen día descubre que lo que cree mejor sabido es en el fondo lo desconocido, ignorado. Por huir de esta ignorancia es por lo que ha nacido [...] (la teoría) y con ello la ciencia". Zubiri, Cinco lecciones de Filosofía, 41. 
convienen todas las cosas porque, al conocer al ente como tal, conoce todo lo que es.

b) Es sophós el que conoce lo más difícil e inaccesible. Pues bien, nada es más difícil e inaccesible que lo universal y la prueba de ello está en la historia misma de la Filosofía, que siempre ha buscado el ente y siempre ha quedado suspendida en la dificultad. Entonces, la ciencia del saber -Filosofía- es suprema sabiduría, dada su dificultad.

c) Se llama sabio al que tiene un saber exacto, estricto y riguroso. El conocimiento apodíctico es el más exacto, estricto y riguroso, puesto que se hace cuestión del nous, principio de todo saber: idea del ente.

d) El sophós sabe algo en forma tan plena que es capaz de enseñarlo. El objeto de la máxima sabiduría es el máximo apto para enseñarlo, porque es universal y, aunque es difícil de aprender, la Filosofía primera es enseñable.

e) El sabio tiene un saber supremo en el sentido de rector, magisterio y mando. A ninguno de los saberes compete con más razón este carácter, ya que todos los demás saberes son parciales; solo la Filosofía primera estudia los principios y las causas supremas. Por lo tanto, las otras ciencias reciben su esclarecimiento y fundamento último de la ciencia apodíctica.

f) La sabiduría es el saber que se busca por sí mismo, por ocio, no con fines prácticos. Para Aristóteles, "cualquier saber y cualquier ciencia es más útil que la filosofía: más noble ninguna”. ${ }^{31}$ Por lo tanto, hay una primacía del saber filosófico sobre los demás saberes.

La Filosofía es la suprema de las ciencias o, dicho de otra manera, es un saber supremo que se basta a sí mismo.

\subsection{La Filosofía como modo de actividad}

Todo saber es un modo ver, un theorem; de allí que toda teoría significa ver algo. Para Aristóteles, la Filosofía es la forma suprema de theoría, es decir, es la forma suprema de ver, es una actividad que consiste en la armonía de la realidad múltiple

31 Aristóteles, Analíticos posteriores, 19. 
y una y no una serie de actos de conocimientos inconexos. En este sentido, actividad significa estar ahí presente, actuando. El filósofo llamó a esta actividad "actualidad". Tal disposición del hombre para actualizarse en algo que tiene sentido en sí mismo y no en algo fuera de él solo es predicable de un ser que sea libre, porque solo tal ser puede disponer de sí mismo, dedicarse al ocio y, de esta manera, además de recrearse en la teoría, le posibilita muchas actividades inéditas, cuya unidad constituye lo que los griegos llamaron vida (bios).

Zubiri interpreta el pensamiento aristotélico y expresa: "Bios es, pues, la unidad de la actividad vital humana; es la energía, la actualidad del viviente humano en su actividad". ${ }^{32}$

La vida, como principio activo o de movimiento actual e inherente al hombre, tiene un fin. La felicidad y la perfección de la vida propia y estrictamente humana, que es la vida según la razón, es la consecuencia del acto perfecto. La vida puede estar dirigida al goce y a la satisfacción personal o bien a la vida política, esto es, a convivir y por último, a la vida teorética o contemplativa, que serían distintos modelos de felicidad. Los dos primeros tipos de bios enunciados necesitan factores externos para lograr su cometido, es decir, no se bastan a sí mismos, sino que requieren de otros y se solventan en virtudes morales.

Véase que la vida política precisa de otro a quien gobernar con justicia y no con malicia; entre tanto, la vida dirigida al goce y a la satisfacción necesita de otros que ayuden a conseguirla. No obstante, estos dos tipos de vida (bios) no agotan las exigencias de felicidad del ser, que es en sí mismo y no en otro; por ello, la vida teorética es la que se corresponde con la máxima felicidad de la persona, por cuanto basta al "sí mismo" mediante un saber que se denomina "virtud(es)".

La vida teorética se caracteriza por una actividad continua de búsqueda de la verdad, de lo que las cosas son. Para Aristóteles, el bios theoretikós o la vida teórica es la más feliz de las vidas, porque su fin (telos) es el más noble, al estar determinado por la parte superior del alma y, dentro de ella, por lo supremo, por el nous. Se atreve a hacer este juicio tras analizar los caracteres que componen este tipo de vida y que resumimos a continuación:

32 Zubiri, Cinco lecciones de Filosofía, 48. 
a) La actividad actual del theorein es superior, en el sentido de que es la más alta, la más fuerte y la más vigorosa en razón de su objeto, que es el nous (conocimiento). Los objetos de este son siempre los más altos y nobles, ya que aprehenden los principios y las causas supremas del universo y no tanto sus resultados prácticos y contingentes.

b) La vida teórica es la más continuada, porque cuando su actividad logra lo que pretende conocer, no se agota; es inagotable como el amor, que no cesa cuando consigue lo que ama, sino que es inacabable.

c) El bios theoretiko es el actuar más autárquico. Solo él se basta a sí mismo. Véase que el sophós sabe por sí mismo, tanto más por sí mismo cuanto más sophós es.

d) Es la única vida amada por sí misma y no por lo que puede suministrar.

e) La felicidad se halla en el ocio y la teoría es mero ocio, en oposición el necocio (negación del ocio o actividad práctica).

\section{Elementos estructurales de la pedagogía para la educación en el Derecho}

La educación es la actividad que consiste en la ayuda que una persona-educadorpresta a otra -educando- para que, por medio de la formación del entendimiento y de la voluntad, pueda ejecutar actos humanos que contribuyan a su perfeccionamiento y al desarrollo de la sociedad. Los actos propios mediante los cuales el educando adelanta su propio proceso de realización se llaman estudio y práctica; el estudio se dirige al cultivo del intelecto y la práctica al cultivo de la voluntad.

El intelecto es el conocimiento de realidad y la práctica es el ejercicio de la realización del bien, que es el objeto de la voluntad; luego, la correcta orientación de las facultades intelectuales y volitivas es tarea prioritaria del educador. Por su parte, a la Pedagogía la constituye el conjunto de estrategias y procedimientos que habrán de emplearse para que el educando alcance los objetos propios del conocimiento de cada ciencia y las habilidades prácticas para su correcto uso y aplicación.

El saber filosófico de la realidad jurídica permite al educando responder correctamente sobre el fundamento del Derecho, su sentido, su estructura y las principales 
corrientes, escuelas y teorías que desarrollan estas cuestiones, para que se ubique de manera pertinente y adecuada en la realidad jurídica de cada tiempo. Para ello, es necesario elaborar un sistema pedagógico que facilite al estudiante la articulación entre el saber teórico -lo especulativo jurídico- y su correspondiente saber hacer en la práctica de su profesión. Este sistema pedagógico es operado entre los sujetos del acto educativo -entre el educador y el educando-, en una relación en la que al educador le corresponde establecer la situación actual del educando frente al estado del conocimiento de la ciencia y al ejercicio práctico de la misma; valorar la capacidad del estudiante para responder a estas de modo eficaz y proactivo; contar con las virtudes humanas necesarias para acompañar al educando en su proceso; tener conocimientos, habilidades y destrezas tanto de la ciencia como de su pedagogía, para trasmitir los contenidos y cumplir con los objetivos del proceso. A manera de resumen, el educador debe tener suficiente conocimiento de su disciplina, claridad mental, rigor, paciencia, amor, confianza y pasión por su ciencia y su discípulo.

Por su parte, el discípulo debe compartir con su maestro el mismo amor por el saber y poseer alto grado de interés por el objeto de estudio, responsabilidad por sus compromisos, una obediencia inteligente, disciplina, perseverancia, optimismo y paciencia.

Visto lo anterior, nos proponemos presentar los elementos de conceptualización fundamentales para definir una metodología en el proceso enseñanza-aprendizaje de la ciencia del Derecho como ciencia práctica.

El ordenamiento jurídico o sistema de Derecho es concebido por Javier Hervada como una estructura ordenadora de la realidad social que, en parte, deviene del derecho natural y, en parte, es creación del hombre. ${ }^{33}$ Para que el orden jurídico sea vivido, requiere su realización con ayuda del jurista, quien está llamado a darle cumplimiento y aplicación. Para que el jurista pueda vivir el ordenamiento jurídico debe conocerlo, es decir, necesita un hábito intelectual específico consistente en saber realizar el orden social justo que la sociedad exige mediante el Derecho. Estudiaremos, entonces, el conocimiento jurídico que le corresponde al jurista con base en las lecciones del profesor citado.

El Derecho, en tanto ordenamiento jurídico, no tiene ningún sentido si no se traduce en una praxis concreta, cuya realización está a cargo del jurista que, a su vez,

33 Hervada, Lecciones propedéuticas, 46. 
debe conocer el propósito de dicho ordenamiento, que no es otro que contribuir al orden social en lo que se refiere a la justicia. En otros términos, al jurista le corresponde la decisión del todo del orden relacional en el aspecto de la justicia, porque el Derecho es una ciencia de carácter práctico.

El carácter práctico del conocimiento del sistema jurídico es un conocimiento intencionalmente dirigido a hacer "algo" y no solo a conocer, que es de lo que trata el conocimiento especulativo. Ese "algo" se traduce en aplicar una norma, cumplir un deber o satisfacer un derecho; en términos generales, en hacer justicia y, por lo tanto, exige de parte del jurista una decisión práctica de la voluntad.

Señala el profesor Hervada que el aspecto formal del saber jurídico, como saber sobre la sociedad humana, es un saber vista sub especie iuris o iustitiae. ${ }^{34}$ En otras palabras, el saber al que nos referimos tiene por objeto de estudio el aspecto jurídico de la sociedad humana, con el fin de contribuir al orden social en razón de los derechos y las deudas u obligaciones correlativas, bien en el orden conmutativo o bien en el orden distributivo.

Este elemento que especifica el saber jurídico tiene diversos grados de conceptualización que se hallan en relación directa con los aspectos aquí enunciados:

1) Fundamental. Epistemológicamente, está determinado porque opera en el plano de abstracción más alto del conocimiento; es el caso de la Ontología Jurídica, que estudia las causas primeras que pueden explicar la realidad jurídica y, en consecuencia, se ocupa de conceptualizar, definir y enunciar de modo propio y distinto acerca del Derecho. Dicho de otra manera, el grado fundamental del conocimiento jurídico es metaempírico, pues se refiere a la esencia misma de la realidad que se nos pone de manifiesto y no se agota con las percepciones experienciales.

2) Científico. Se caracteriza por un conocimiento fenoménico y experimental y, por tanto, se pregunta por las causas próximas, por los fenómenos captables empíricamente, por ejemplo, la ley promulgada, la sentencia o el contrato, las costumbres, los usos sociales, el Derecho conciliado, los acuerdos.

34 Hervada, Lecciones propedéuticas, 595. 
3) Casuismo. Se propone sintetizar las conclusiones derivadas de los grados anteriores, con el fin de resolver posibles casos pasados, presentes o posibles a modo ejemplar. No se propone analizar teóricamente la realidad jurídica como lo hacen los dos primeros niveles mencionados, por ejemplo, la jurisprudencia, teoría del caso, análisis de decisiones judiciales, criterios de interpretación y ponderación del juicio.

4) Prudencial. Se refiere a la decisión inmediata, práctica y existencial con la cual se realiza el orden jurídico en la vida real. Es producto de la virtud de la prudencia que se adquiere del conocimiento de los anteriores grados y se perfecciona por la decisión real de la voluntad de su compromiso con la obra justa. Esto significa que si en la decisión no existe la voluntad definida de realizar primariamente una obra justa, dicho conocimiento práctico queda incompleto, pues este es el que perfecciona el acto jurídico y compromete la eficacia de todo el ordenamiento jurídico.

De acuerdo con lo anterior, el conocimiento jurídico es un conocimiento práctico en el sentido más estricto del término, ya que integra el conocimiento teórico por medio de los dos primeros y el técnico, de los dos últimos.

Conviene anotar que cada grado es diferenciable, pues maneja una peculiar forma de conceptualización y, por lo tanto, es autónomo:; 35 in embargo, ninguno puede considerarse exclusivo, ya que todos son insuficientes para conocer y realizar el orden jurídico en su totalidad, ya que cada uno ofrece una visión de la verdad sin agotarla, de lo que se deriva la necesidad de una conexión entre ellos al ser un mismo objeto conocido. Señala el profesor Hervada que hay que evitar dos defectos: "[... ] primeramente la confusión metodológica y el uso indiscriminado por una ciencia de los conceptos y resultados elaborados por otra; en segundo lugar, convertir la autonomía de los distintos niveles en autosuficiencia, propugnando o siguiendo una pureza metódica total". ${ }^{36}$

\footnotetext{
35 El profesor Hervada señala: "Por autonomía científica se entiende que cada ciencia en particular (cada nivel) tiene un método propio y un aparato conceptual específico, correspondientes a una perspectiva formal o modo de sintonizar intelectualmente con la realidad jurídica”. Hervada, Lecciones propedéuticas, 599.

36 Hervada, Lecciones propedéuticas, 601.
} 


\section{Conclusiones}

El pensamiento filosófico, en particular el pensamiento filosófico-jurídico, es un saber que ha acompañado al ser humano desde su aparición y ha sido determinante en su proceso de acercamiento a la fundamentación y al sentido de la realidad jurídica (dimensión noética), lo que ha supuesto una apertura a esa realidad para inquirir y establecer paulatinamente sus causas. Luego, hacerse cargo de la realidad jurídica y advertirla como un débito humano es hacerse cargo de la dimensión ética y, a la vez, el compromiso que esta dimensión ética establece con la obra humana es, en últimas, encargarse de la dimensión práctica (dimensión práxica).

Mostrar "lo jurídico" como realidad humana, para llegar a conocerla y aceptarla como condición del débito, para de allí reconocerla como condición sine qua non del acto humano de justicia, es una labor que le corresponde a los profesores formados en este campo del saber y que tienen a su cargo el grave compromiso de enseñar la Filosofía del Derecho.

En la formación en Filosofía del Derecho no debe olvidarse el quehacer del jurista. Con ese fin, ha de ser dueño de un saber pedagógico que le permita definir su método de trabajo en el aula y fuera de ella para estar en las dimensiones expuestas, ya que el pensamiento jurídico, desde sus causas y saberes, permea constantemente a todos los seres humanos y a las sociedades que conforman e influye a las personas en su quehacer cotidiano.

Por lo anterior, podríamos decir que el pensamiento filosófico sigue teniendo vigencia hoy más que nunca en la calidad de vida, caracterizada por el reconocimiento de la dimensión jurídica de la persona humana y de esta como núcleo del todo social.

\section{Referencias}

Aristóteles. Analíticos posteriores. Madrid: Gredos, 1994.

Aristóteles. Metafísica. Madrid: Gredos, 1994.

Copleston, Frederick. Historia de la Filosofia 1: Grecia y Roma. Barcelona: Ariel SA, 1999.

Gran enciclopedia Rialp. "Realidad". Madrid: Rialp, 1989.

Gran enciclopedia Rialp. "Realismo". Madrid: Rialp, 1989. 
Hervada, Javier. Lecciones propedéuticas de Filosofía del Derecho. 3a edición. Pamplona: Eunsa, 2000.

Martínez García, Enrique. Persona y educación en santo Tomás de Aquino. Madrid: Fundación Universitaria Española, 2002.

Tomás de Aquino. Suma de Teología. parte II. 166: Templanza. La estudiosidad. Madrid: Biblioteca de autores cristianos, 2001.

Universidad Católica de Colombia. "Derecho". https://www.ucatolica.edu.co/portal/programas/programas-de-pregrado/derecho/ (acceso octubre 25, 2015).

Yepes Stork, Ricardo y Javier Aranguren. Fundamentos de Antropología: un ideal de la excelencia humana. Pamplona: Eunsa, 1998.

Zubiri, Xavier. Cinco lecciones de Filosofía. Madrid: Alianza, 1982. 